\title{
Simultaneous Gastric and Ileal Trichobezoars Causing Small Bowel Obstruction: A Case report
}

\author{
Lal S', Shukla $\mathbf{P}^{2}$, Yedalvar V', Shrivastava GP ${ }^{4}$
}

${ }^{1}$ Dr. S Lal, MBBS, MS, Assistant Professor, ${ }^{2}$ Dr. P Shukla, MBBS, MS, Assistant Professor, ${ }^{3}$ Dr. V Yedalvar, MBBS, MS, Associate Professor, ${ }^{4} \mathrm{Dr}$. GP Shrivastava, MBBS, MS, Professor and Head of Department. All from the Department of Surgery, Shyam Shah Medical College and Associated SGM Hospital Rewa, Madhyapradesh, India.

Adddress for correspondence: Dr. S. Lal, E-mail: slaldr@gmail.com

\begin{abstract}
Bezoars are conglomerates of food or fiber in the alimentary tract of humans and certain animals, mainly ruminants. A trichobezoar represents a mass of accumulated hair. Trichobezoars may present as an isolated gastric mass, as an extension into the small intestine, or as an independent fragmented mass in the small intestine. The presence of discrete coexisting gastric and ileal trichobezoars has been reported only rarely in the literature. This is a case report of a 10-year-old girl presenting with small-bowel obstruction secondary to synchronous trichobezoars in the stomach and ileum. The case highlights the role of imaging and importance of complete evaluation of the gastrointestinal tract at the time of surgical evacuation.
\end{abstract}

Key words: Trichobezoars, stomach, small bowel, obstructions

\section{Introduction}

B ezoars are foreign bodies in the gastrointestinal tract that increase in size by accretion of nonabsorbable food or fibers. Lactobezoars (milk or formula), phytobezoars (plant fiber), pharmacobezoars (pills or capsules), and trichobezoars (hair) comprise the four types. In children and adolescents, trichobezoars are most prevalent and usually result from trichotillomania (a behavioral disorder characterized by the compulsive urge to pull one's hair) combined with trichophagia (the ingestion of hair). Trichobezoars can rarely cause smallbowel obstruction. We describe a case of a 10-year-old girl with synchronous gastric and obstructing smallbowel bezoars and review the clinical presentation, imaging findings, and surgical management.

\section{Case Report}

A 10-year-old girl presented to the emergency surgery department with a 3-day history of crampy abdominal pain and bilious vomiting. She had complained of epigastric lump since last one year. Her medical history was significant for trichotillomania in early childhood, which her parents felt had resolved at 4 years of age. On physical examination, the patient's hair was normal with no visible alopecia. The abdomen was tender and associated with peritoneal signs. Laboratory investigations showed leucocytosis. A plain abdominal $X$-ray showed dilated small bowel loops with no free air. On ultrasound two separate masses; one adjacent to left lobe of liver and another in small -bowel was seen. Given the history of trichophagia, a provisional diagnosis of trichobezoar with intestinal obstruction was made. An exploratory laparotomy through an upper midline abdominal incision was performed.

The stomach was distended and containing a large mobile mass within the gastric lumen. A gastrotomy was made over the body of the stomach, and a $15 \times 6 \times 4-\mathrm{cm}$ trichobezoar was extracted (Fig. 1).

There was no extension of hair into the duodenum. One independent mass was palpated in the terminal ileum which was extracted through entrotomy. It measured $5 \times 3.5 \times 2.4 \mathrm{~cm}$ (Fig. 2). 
The child recovered uneventfully. Postoperatively psychiatric evaluation showed that patient psychologically stable and did not reveal any evidence of anxiety or depression.

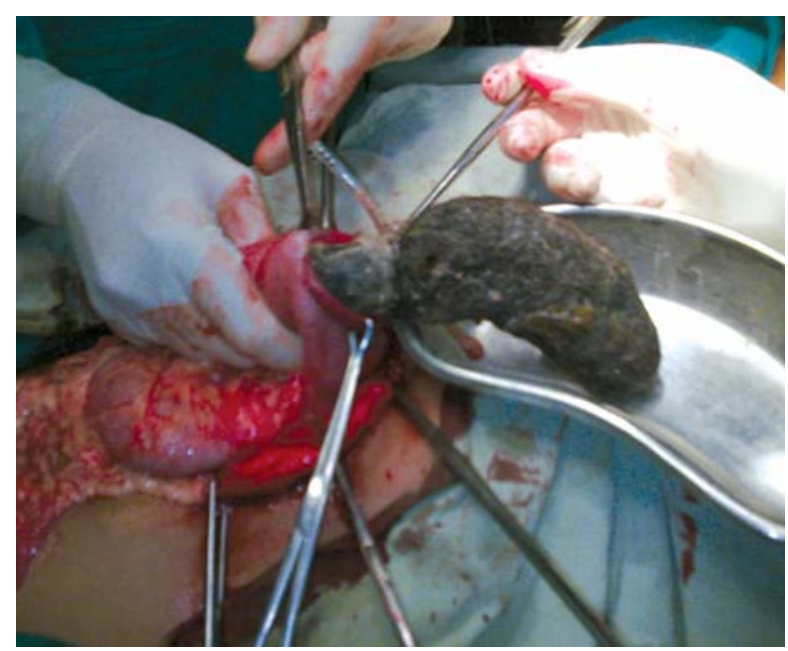

Fig 1: The large trichobezoar being extracted through the gastrotomy.

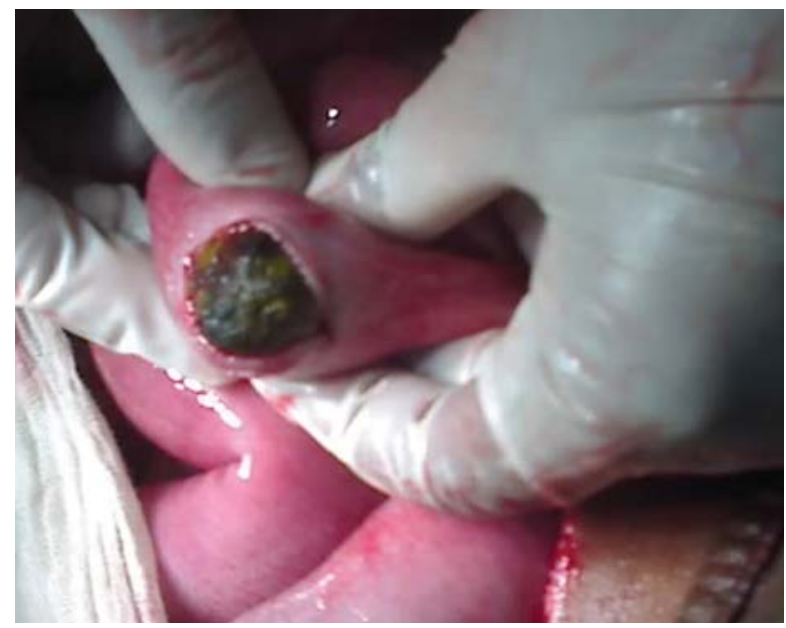

Fig 2: Peroperative photograph showing removal of trichobezoar through entrotomy.

\section{Discussion}

Bezoars result from ingestion of foreign material that accumulates in the gastrointestinal tract because of large particle size, indigestibility, gastric outlet obstruction, or intestinal stasis. The term bezoars derives from the Arabic word Badzehr, which means antidote. Bezoars were used as antidotes and as magical medicinal agents against plague, snake-bite, sexual weakness, leprosy and epilepsy by physician from $12^{\text {th }}$ to $18^{\text {th }}$ century.

In the classic review by DeBakey and Ochsner ${ }^{1}$, $80 \%$ of trichobezoars were found in patients younger than 30 years. Trichobezoars are located typically in the stomach, and occur primarily in girls who chew and swallow their own hair. Although about 1 of 2000 children suffers from trichotillomania, only half of the patients have shown a history of trichophagia ${ }^{2}$ and just $1 \%$ of those individuals eat enough hair to accumulate trichobezoars that require surgical intervention. The hair fibers become trapped in the gastric mucosal folds, and gastric acid denatures hair proteins and blackens the bezoar, regardless of the intrinsic color. Factors associated with trichobezoar formation include developmental delay, female sex, and an underlying behavioral disorder leading to pica and compulsive ingestion of food and nonfood substances. Symptoms are nonspecific and may include midepigastric pain, failure to thrive, early satiety, nausea and vomiting, constipation, or diarrhoea.

In most cases, trichobezoars are present only in the stomach. Five percent of gastric bezoars are multiple. Fifteen percent of trichobezoars are intestinal. The term Rapunzel syndrome was given to trichobezoars extending continuously through the entire length of the small intestine as a tail and was first described by Vaughan et $\mathrm{al}^{3}$ in 1968. Small-bowel obstruction from a trichobezoar is very uncommon. Because trichobezoars do not usually migrate into the small bowel, the presence of simultaneous gastric and small intestinal bezoars has been rarely reported ${ }^{4-6}$.

Various imaging modalities have been recommended for detection of bezoars. The conventional radiography shows a mass of opaque soft tissue in a swollen stomach and can reveal bowel obstruction with dilated small bowel loops with fluid and air filed loops. A calcified rim may delineate the edge of the bezoar. Both, contrast radiography and the endoscopy for establishing the diagnosis of the upper GI tract are the diagnostic procedure of choice. On CT, a bezoar is an intraluminal low-attenuation mass with a characteristic mottled and heterogeneous appearance; sometimes containing trapped air ${ }^{7}$. Orally administered contrast material can delineate its outer surface. On ultrasound, a bezoar will typically present as a curvilinear bright echogenic band caused by the presence of a ball of hair and multiple acoustic interfaces created by trapped air and food. Recently, MRI recommended which show the bezoars as a mass in the small bowel containing mottled and confluent low signal intensities on both T1- andT2- weighted MR images ${ }^{8}$. Upper endoscopy, in addition to being a diagnostic aid for trichobezoar, may be used for therapeutic retrieval of small proximal trichobezoars $^{9}$. Endoscopic fragmentation and retrieval of bezoars, with or without enzymatic dissolution, are technically challenging. Early surgical intervention is the only satisfactory treatment of large or complicated 
trichobezoars. Bezoars may be removed either laparoscopically ${ }^{10}$ or by open laparotomy. Due to $100 \%$ success rate, the relatively low complexity, and the ability to carefully examine the entire gastrointestinal tract for satellites in a short period of time, laparotomy is still considered the treatment of choice.

\section{Conclusion}

Trichobezoars are rare clinical entity. Stomach is the common site of occurrence. Primary intestinal trichobezoar are uncommon. The presence of discrete coexisting gastric and ileal trichobezoars are extremely rare. Various imaging modalities have been recommended for diagnosis. As far as treatment is concerned, however, we consider conventional laparotomy to be the treatment of choice. It is mandatory to perform a thorough exploration of all the small intestine and the stomach searching for retained bezoars. Psychiatric evaluation and follow-up are essential to treat any underlying behavioral illness that predisposes to recurrence.

\section{References}

1. DeBakey M, Ochsner A. Bezoars and concretions: a comprehensive review of the literature with an analysis of 303 collected cases and 8 additional cases. Surgery. 1938; 4:934-963.

2. Yao CC, Wong HH, Chen CC, Wang CC, Yang CC, Lin. CS. Laparoscopic Removal of Large Gastric Phytobezoars. Surg Laparosc Endosc Percutan Tech 2000; 10: 243-5.
3. Vaughan ED, Sawyers JL, Scott HW. The Rapunzel syndrome. An unusual complication of intestinal bezoar. Surgery. 1968;63:339-343.]

4. Malhotra A, Jones L, Drugas G. Simultaneous gastric and small intestinal trichobezoars. Pediatr Emerg Care. 2008 249(11):774-776.

5. Hoover K, Piotrowski J, St Pierre K, Katz A, Goidstein AM. Simultaneous gastric and small intestinal trichobezoars- a hairy problem. J Pediatr Surg. 2006 41(8):1495-7.

6. Kovács V, Bruncák $P$, Cseri J, Malatinec J.Simultaneous bezoars in the stomach, ileum and cecum causing acute ileus in a 30-year-old imbecile-case report. Rozhl Chir 2002;81:248-51.

7. Billaud Y, Pilleul F, Valette PJ, Mechanical small bowel obstruction due to bezoars: Correlation between CT and surgical findings. J Radiol 2002; 83:641-6.

8. Lee JM, Jung SE, Lee KY.Small -bowel obstruction caused by phytobezoar: MR imaging findings. AJR Am J Roentgen 2002; 179:538-9.

9. Alsafwah S, Alzein M. Small bowel obstruction due to trichobezoar: role of upper endoscopy in diagnosis. Gastrointest Endosc. 2000; 52: 784786.

10. Palanivelu C, Rangrajan M, Senthilkumar R, Madankumar M V. Trichobezoars in the stomach and ileum and their laproscopy-assisted removal: a bizarre case. Singapore Med J 2007; 48(2):e37-39.

\section{How to cite this article?}

Lal S, Shukla P, Yedalvar V, Shrivastava GP. Simultaneous Gastric and Ileal Trichobezoars Causing Small Bowel Obstruction: A Case report. J Nepal Paedtr Soc 2011;31(1):72-74. 